\title{
Compliance of General Health Clinics to National Licensure Standards: A Survey in Northwestern Iran
}

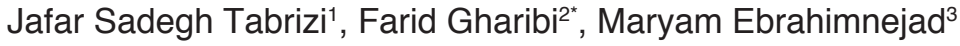 \\ ${ }^{1}$ Health Services Management Research Center, School of Management and Medical Informatics, Tabriz University \\ of Medical Sciences, Tabriz, Iran. ${ }^{2}$ Students' Research Committee, School of Management and Medical Informatics, \\ Tabriz University of Medical Sciences, Tabriz, Iran. ${ }^{3}$ School of Management and Medical Informatics, Tabriz University \\ of Medical Sciences, Tabriz, Iran.
}

First Published online March 30, 2016

\begin{abstract}
Background and Objectives: Monitoring of health organizations by the regulatory bodies is crucial to ensure high performance of health system. Although Iranian health settings are monitored by the Ministry of Health and Medical Education (MOHME) in various ways, periodic evaluation of their constant commitment to the national licensure standards in health domain is rare. The aim of the present study was to highlight the importance of such evaluations by auditing the general health clinics of Tabriz (a metropolitan city in Northwestern Iran) based on national licensure standards.
\end{abstract}

Methods: This across-sectional study surveyed all general health settings in Tabriz city. The data were collected using a validated researcher-made checklist. The collected data were summarized using descriptive statistical methods. The mean values were compared by $t$ test and analysis of variance (ANOVA).

Findings: The score of compliance to national licensure standards averaged at $70.5 \%$. The highest level of compliance was observed for medical and non-medical devices dimension (78.2\%) whereas the lowest compliance was recorded for hygiene dimension (58.4\%). Public clinics showed a significantly higher compliance to standards compared with the private settings $(P<0.05)$. Also a significant difference in total compliance score was identified between clinics with $>$ and $<5$ licensed services $(P<0.05)$.

Conclusions: The level of current compliance of the surveyed clinical settings shows considerable room for improvement. Private health settings and the hygiene domain are the primary targets of focus for intervention. Our results encourage further national-wide studies to gain a comprehensive view of the compliance of Iranian clinics to the licensure standards.

Keywords: Licensure, Hospital, Benchmarking, Clinical audit, Health system

\section{Background and Objectives}

Monitoring of health organizations by the regulatory bodies is crucial to ensure high performance of health system and enabling managers and policy makers for evidence-based decision making. ${ }^{1-3}$ There are various approaches to evaluate and ensure the quality of services delivered by health organizations, including certification, accreditation, and licensure. ${ }^{4-6}$ Licensure in health domain is referred to as granting license by an official or legal body to an organization to provide a specific health service according to the standards of the reference body and

*Corresponding Author: Farid Gharibi, Students' Research Committee, School of Management and Medical Informatics, Tabriz University of Medical Sciences, Tabriz, Iran. Postal Code: 5166614711, Tel: +98918 133 2935, Fax: +98 4133352291, Email: gharibihsa@gmail.com the same quality. ${ }^{4}$ The purpose of licensure is to support patients in receiving safe and quality health services and to alleviate the health risk of unauthorized or non-standards medical practices. ${ }^{7,8}$

In Iran, licensed health organizations are usually audited before receiving a license for health services. Nevertheless, there is little evidence to show that the compliance of the licensed health settings to the licenser's standards is constantly monitored..$^{9-12}$ This fact together with other relevant shortcomings such as lack of valid standards, reliance on subjective evaluation, lack of an appropriate evaluation process and data collection mechanisms, and inadequate training of surveyor, limits the efficiency of health services monitoring system for ensuring 
quality of health services. One of the major health fields for appearance of these shortcomings is the outpatient care which account for the majority of patients' referrals. ${ }^{13,14}$

Survey of literature in this domain revealed a gap in relevant studies and reports in an Iranian context, maintaining the situation of Iranian health clinics totally vague. This gap motivated us to conduct a study aimed to (1) develop a validated evaluation tool for regular assessment of the commitment of health clinics to the licensure standards, and (2) to provide relevant provincial-level data as a baseline for further large-scale surveys.

\section{Methods}

Of the total of 65 general clinics of Tabriz city (Northwestern Iran) 49 clinics whose administrators agreed to participate in the study were surveyed. Given the unavailability of standard tools to assess compliance with licensure standards, researchers developed and validated a checklist for this purpose (see Supplementary Materials).

The checklist questions were initially formulated based on extensive literature review and the opinion of 10 experts from Treatment Affairs of Tabriz University of Medical Sciences, Tabriz Medical Council, and Tabriz Health Services Management Department. The content validity of the checklist was confirmed using content validity index $(\mathrm{CVI})$ and the content validity ratio (CVR) which was tested by mentioned experts. An acceptance score of $70 \%$ was calculated based on the experts' responds. ${ }^{15,16}$ Each item below this score was set to be eliminated from the checklist. The CVR and CVI of $89 \%$ and $92 \%$, respectively, were obtained without excluding any questions. The final tool was a 78-item checklist with six dimensions of physical environment (23 questions), medical and non-medical devices (16 questions), human resources (13 questions), medical documents and information (5 questions), compliance the law and regulations notified (10 questions) and hygiene (11 questions). In addition, some questions regarding the characteristics of health clinics, including geographical region, date of launch, admission rate, ownership, and the number of approved services delivered are included in checklist.

The data was collected by interviewing clinical managers and staff, field observations, and review of clinical documents/records based on the nature of checklist questions. Data was summarized using descriptive statistical methods. $T$ test and analysis of variance (ANOVA) was used to compare the mean values. All analyses were carried out by SPSS version 19 software package.

Ethical Issues

This study was approved by Ethics Committee of Tabriz
University of Medical Sciences. The informed consent of all participants was obtained. The participants were insured of the confidentiality of their responses.

\section{Results}

Table 1 shows the characteristics of the surveyed hospitals. The majority of the surveyed clinics hold a private ownership (71.5\%) and work entire day and night (87.8\%). The admission rate in clinics ranges between 50-100 patients/day. Most clinics (51\%) are authorized to offer 6-10 approved health services (Table 1).

Table 2 presents the score mean of compliance with licensure standards and its dimensions. The compliance with licensure standards averaged $70.5 \%$. The highest score mean was received by dimension of medical and non-medical devices (78.2\%) and the score was related to dimension of hygiene (58.4\%).

$T$ test revealed that the overall compliance and three dimensions of physical environment, medical and non-medical devices, and human resources score significantly higher in public compared with private clinics $(P<0.05)$. Also ANOVA identified significantly higher compliance in clinics offering $>15$ approved services compared with those offering $1-5(P<0.5)$.

\section{Discussion}

The present study aimed to audit the general health clinics of Tabriz city with respect to their compliance to the licensure standards. The surveyed clinics showed a moderate compliance with the licensure standards. A previous similar study by Ahmadi et al identified a compliance score of $78.9 \%$ for general practitioners offices (GPOs) of Tabriz city. ${ }^{17} \mathrm{~A}$ comparable score of $78.5 \%$ was also reported by Kamkari et al, for Tabriz's general dentistry offices

Table 1. The Characteristics of Assessed Clinics

\begin{tabular}{lccc}
\hline Variables & Categories & No. & $\%$ \\
\hline \multirow{2}{*}{ Ownership } & Public & 12 & 24.5 \\
& Private & 35 & 71.4 \\
& Charity & 2 & 4.1 \\
\hline \multirow{2}{*}{ Time of services delivery } & Daily (part-time) & 6 & 12.2 \\
& 24 hours & 43 & 87.8 \\
\hline \multirow{3}{*}{ Admission rate } & $1-50$ & 8 & 16.3 \\
& $51-100$ & 16 & 32.7 \\
& $101-200$ & 11 & 22.4 \\
& $>200$ & 14 & 28.6 \\
\hline \multirow{2}{*}{ No. of approved services } & $1-5$ & 17 & 34.7 \\
delivered & $6-10$ & 25 & 51.0 \\
& $11-15$ & 4 & 8.2 \\
\hline
\end{tabular}


Table 2. The Status of Standards Compliance in All Dimensions

\begin{tabular}{lll}
\hline Dimension & Mean & SD \\
\hline Physical environment & 0.75 & 0.17 \\
Medical and non-medical devices & 0.78 & 0.12 \\
Human resources & 0.65 & 0.13 \\
Medical documents and informatics & 0.61 & 0.21 \\
Compliance of law and notified regulations & 0.69 & 0.20 \\
Hygiene & 0.58 & 0.18 \\
Total (all of dimensions and standards) & 0.705 & 0.123
\end{tabular}

Abbreviation: SD, standard deviation.

(GDOs). ${ }^{18}$ The lower compliance of general health clinics in the present survey compared with GPOs and GDOs may be attributed to the apparently more competitive nature of activities in offices compared with clinics.

The highest compliance score was recorded for medical and non-medical devices. This may be due to the positive attitude of patients towards the advanced medical/ non-medical equipment and the efforts made by clinical administrators to take the advantage of this attitude to attract more patients. On the other hands, and perhaps as a result of over-emphasis on equipment, such an important factor as hygiene scores the lowest. The marginal attention of clinics to the hygiene was also reported in the study of Ahmadi et al where hygiene received the lowest compliance score in GPOs. ${ }^{17}$

With the exception of a few domains, there is no agreement between findings of our and previous similar studies carried out in Tabriz. For instance contrary to our results, Ahmadi et $\mathrm{al}^{17}$ and Kamkari et $\mathrm{al}^{18}$ found the highest score mean for the dimension of medical documents and informatics. Also in contrast to our data, the dimension of medical and non-medical devices scored the lowest in the survey of dentistry offices by Kamkari et al. ${ }^{18}$ These discrepancies indicate that the adherence to licensure standards may differ depending on the medical context, thus requiring context-specific intervention approaches.

We also found significantly higher score of compliance to standards in clinics with more than 15 approved services compared with those with lower than 5 . This signals a correlation between the level of compliance and number of licensed services and compliance with licensure standards, which if confirmed in future studies would be informative for policy-making and intervention.

A higher level of compliance with standards was also found in public clinics compared with the private clinics. This difference does not follow the apparently more positive attitude of patients towards private health settings relative to the public ones. Investigating the reason behind this difference is beyond the scope of this study. Our results however emphasize the need for such studies to gain insight into the impact of ownership on compliance of clinics to the standards and the required interventions to improve the situation in private settings.

\section{Conclusions}

The level of current compliance of the surveyed clinical settings shows considerable room for improvement. Private health settings and the hygiene domain are the primary targets of focus for intervention. Our results encourage further national-wide studies to gain a comprehensive view of the compliance of Iranian clinics to the licensure standards.

\section{Abbreviations \\ (GP): general practitioner; (CVR): content validity ratio; (CVI): content validity index; (GDOs): general dentistry offices.}

\section{Authors' Contributions}

JST designed the study and contributed to drafting the manuscript. FG was involved in analyzing and interpreting the data and drafting the manuscript. ME took part in literature review, data collection and analysis. All authors read and approved the final manuscript.

\section{Competing Interests}

The authors declare no competing interests.

\section{Acknowledgments}

The authors appreciate collaboration of personnel of the surveyed health clinics and financial support of Tabriz's Health Services Management Research Centers.

\section{Supplementary Materials}

Supplementary file contains the assessment checklist.

\section{References}

1. Shaw CD. The external assessment of health services. World Hosp Health Serv. 2004;40(1):24-28.

2. Shaw CD. Toolkit for Accreditation Programs. Melborne: International Society for Quality In health Care (ISQua); 2006.

3. Mert T, Ekici D. Development of an Assessment Model for Evaluating the Performance of Nursing Services. Int J Hosp Res. 2015;4(1):9-14.

4. Rooney AL, Van Ostenberg PR. Licensure, Accreditation, and Certification: Approaches to Health Services Quality. Center for Human Services (CHS); 1999.

5. Saadati M, Yarifard K, Azami-Agdash S, Tabrizi JS. Challenges and Potential Drivers of Accreditation in Iranian 
Hospitals. Int J Hosp Res. 2015;4(1):37-42.

6. Rahimi H, Khammar-Nia M, Kavosi Z, Eslahi M. Indicators of Hospital Performance Evaluation: A Systematic Review. Int J Hosp Res. 2014;3(4):199-208.

7. Saufl NM, Fieldus MH. Accreditation: a "voluntary" regulatory requirement. J Perianesth Nurs. 2003 Jun;18(3):152-9. doi:10.1016/s1089-9472(03)00087-x.

8. Tabrizi JS, Gharibi F. Systematic survey of accreditation models for designing a national model. Scientific Journal of Kurdistan University of Medical Sciences. 2011;16(3):95109.

9. Tabrizi JS, Gharibi F, Pirahary S. Developing of national accreditation model for rural health centers in Iran health system. Iran J Publ Health. 2013;42(12):1438-1445.

10. Safdari R, Meidani S. Health Services Accreditation Standards for information management in Canada, New Zealand and USA: a comparative study. J Qazvin Univ Med Sci. 2007;11(1):73-78.

11. Golamzade Nikjou R, Dadgar E, Baqeri S, Gharibi F, Mojahed F, Kalantari H. Analyzing of evaluation tools and process in Tabriz hospitals emergency departments. Depiction of Health. 2013;4(1):35-41.

12. Vatankhah S, Salemi A. The review of Iran university-led hospitals' assessment system, by BSC method. J Health Manag. 2008;38(12):50-53.

13. Tabrizi JS, Gharibi F, Ramezani M. Development of a national accreditation model in specialized clinics of hospitals. Hakim Res J. 2012;15(3):229-237.

14. Abdolahi R. The Principles of Supervision on Ambulatory Care Centers. Tehran: Pouneh Publisher; 2010.

15. Yaghmaie F. Content validity and its estimation. J Med Educ. 2003;3(1):25-27

16. Hajizadeh E, Asghari M. Statistical Methods and Analyses in Health Biosciences. Tehran: Jahad Daneshgahi Publisher; 2010.

17. Ahmadi F, Tabrizi JS, Gharibi F. The Auditing of General Practitioner Offices in Tabriz City to Assess Their Compliance With National Licensure Standards in 2014. Tabriz: School of Health Management and Medical Informatics, Tabriz University of Medical Sciences; 2015.

18. Kamkari M, Tabrizi JS, Gharibi F. The Auditing of Tabriz General Dentistry Offices to Assess their Compliance With National Licensure Standards, 2015. Tabriz: School of Health Management and Medical Informatics, Tabriz University of Medical Sciences; 2015.

\section{Please cite this article as:}

Tabrizi JS, Gharibi F, Ebrahimnejad M. Compliance of general health clinics to national licensure standards: a survey in Northwestern Iran. Int J Hosp Res. 2016;5(1):13-16. doi:10.15171/ijhr.2016.03. 